\title{
Hereditary Early-Onset Parkinson's Disease Caused by Mutations in PINK1
}

Enza Maria Valente, ${ }^{1 * \dagger}$ Patrick M. Abou-Sleiman, ${ }^{2 *}$ Viviana Caputo, ${ }^{1,3 \dagger}$ Miratul M. K. Muqit, ${ }^{2,4 \dagger}$ Kirsten Harvey, ${ }^{5}$ Suzana Gispert, ${ }^{6}$ Zeeshan Ali, ${ }^{6}$ Domenico Del Turco, ${ }^{7}$ Anna Rita Bentivoglio, ${ }^{8}$ Daniel G Healy, ${ }^{2}$ Alberto Albanese, ${ }^{9}$ Robert Nussbaum, ${ }^{10}$ Rafael González-Maldonado, ${ }^{11}$ Thomas Deller, ${ }^{7}$ Sergio Salvi,${ }^{1}$ Pietro Cortelli, ${ }^{12}$ William P. Gilks, ${ }^{2}$ David S. Latchman, ${ }^{4,13}$ Robert J. Harvey, ${ }^{5}$ Bruno Dallapiccola, ${ }^{1,3}$ Georg Auburger, ${ }^{14 \div}$ Nicholas W. Wood ${ }^{2 !}$

${ }^{1}$ CSS IRCCS, Mendel Institute, viale Regina Margherita 261, 00198 Rome, Italy. ${ }^{2}$ Department of Molecular Neuroscience, Institute of Neurology, Queen Square, London, WC1N 3BG, UK. ${ }^{3}$ Department of Experimental Medicine and Pathology, University La Sapienza, Viale Regina Elena 324, 00187 Rome, Italy. ${ }^{4}$ Medical Molecular Biology Unit, Institute of Child Health, 30 Guilford Street, London WC1N 1EH, UK. ${ }^{5}$ Department of Pharmacology, The School of Pharmacy, 29/39 Brunswick Square, London WC1N 1AX, UK. ${ }^{6}$ Institute for Experimental Neurobiology, J.W. Goethe University, Theodor Stern Kai 7, 60590 Frankfurt/M, Germany. ${ }^{7}$ Institute of Clinical Neuroanatomy, J.W. Goethe University, Theodor Stern Kai 7, 60590 Frankfurt/M, Germany. ${ }^{8}$ Institute of Neurology, Catholic University, largo A. Gemelli 8, I-00168 Rome, Italy. ${ }^{9}$ National Neurologic Institute Carlo Besta, via Celoria 11, 20133 Milan, Italy. ${ }^{10}$ National Human Genetics Research Institute, National Institutes of Health, 49 Convent Drive, Bethesda, MD 20892, USA. ${ }^{11}$ Department of Neurology, Hospital Universitatio San Cecilio, Avenida Dr. Olóriz s/n, 18012 Granada, Spain. ${ }^{12}$ Department of Neurosciences, University of Modena and Reggio Emilia, via del Pozzo 71, 41100 Modena, Italy. ${ }^{13}$ Birkbeck, University of London, Malet Street, London WC1E 7HX, UK. ${ }^{14}$ Section Molecular Neurogenetics, Clinic for Neurology, J.W. Goethe University, Theodor Stern Kai 7, 60590 Frankfurt/M, Germany.

*These authors contributed equally to this work.

$\dagger$ These authors share joint second authorship.

†To whom correspondence should be addressed. E-mail: n.wood@ion.ucl.ac.uk (N.W.W.); auburger@em.uni-frankfurt.de (G.A.); e.valente@css-mendel.it (E.M.V.)

Parkinson's disease (PD) is a neurodegenerative disorder characterized by degeneration of dopaminergic neurons in the substantia nigra. We previously mapped a locus for a rare familial form of PD to chromosome 1 p36 (PARK6). Here we show that mutations in PINK1 (PTEN-induced kinase 1), are associated with PARK6. We have identified two homozygous mutations affecting the PINK1 kinase domain in three consanguineous PARK6 families, a truncating nonsense mutation and a missense mutation at a highly conserved amino acid. Cell culture studies suggest that PINK1 is mitochondrially located and may exert a protective effect on the cell which is abrogated by the mutations, resulting in increased susceptibility to cellular stress. These data provide a direct molecular link between mitochondria and the pathogenesis of PD.

Parkinson's disease (PD) is a common neurodegenerative disorder that is characterized by loss of dopaminergic neurons in the substantia nigra and the presence of cytoplasmic protein inclusions known as Lewy bodies. The majority of PD cases are sporadic; however, the identification of a number of genes responsible for rare familial forms of PD has provided important insights into the underlying mechanisms of the disease. These genes, encoding $\alpha$-synuclein, parkin, UCH-L1 and DJ-1 have implicated protein misfolding, impairment of the ubiquitin-proteasome system, and oxidative stress in the pathogenesis of the disease $(1,2)$.

We previously mapped PARK6, a locus linked to autosomal recessive, early-onset $\mathrm{PD}$ to a $12.5 \mathrm{cM}$ region on chromosome 1p35-p36 by autozygosity mapping in a large consanguineous family from Sicily (3). Subsequent identification of two additional consanguineous families (one from Central Italy (family IT-GR) (4) and one from Spain) provided additional evidence of linkage to PARK6. A critical recombination event in the Spanish family refined the candidate region to a $3.7 \mathrm{cM}$ interval between flanking markers D1S2647 and D1S1539. Fine mapping of single nucleotide polymorphisms and newly generated short tandem repeat markers in the three families defined a $2.8 \mathrm{Mb}$ region of homozygosity within contig NT_004610, containing approximately 40 genes.

Candidate genes were prioritized on the basis of their putative function and expression in the central nervous system, as assessed by bioinformatic analysis and by exon amplification from a human substantia nigra cDNA library. 
Sequence analysis of candidate genes in affected members from each family led to the identification of two homozygous mutations in the PTEN- induced putative kinase 1 (PINK1) gene. The mutations segregated with the disease phenotype in the three consanguineous families, were confirmed in the cDNA, and were absent from 400 control chromosomes including 200 chromosomes from Sicilian individuals. The Spanish family carried a G>A transition in exon 4 (nt 11185 in (NT_004610)) resulting in the substitution of a highly conserved amino acid in the putative kinase domain, G309D (fig. S1). Both Italian families carried the same G>A transitions in exon 7 (nt 15600 in (NT_004610)), which results in a W437OPA substitution, truncating the last 145 amino acids encoding the $\mathrm{C}$-terminus of the kinase domain. These families shared a common haplotype, implying common ancestry (table $\mathrm{S} 1$ ).

The PINK1 gene contains eight exons spanning $\sim 1.8 \mathrm{~Kb}$ and encodes a 581 amino acid protein. The transcript is ubiquitously expressed (5) and is predicted to encode a 34amino acid mitochondrial targeting motif (cleavage site for the mitochondrial processing peptidase between residues 34 and 35) and a highly conserved protein kinase domain (residues 156 to 509) that shows high degree of homology to the serine/threonine kinases of the $\mathrm{Ca}^{2+} /$ calmodulin family. To investigate the consequences of the missense mutation at the cellular level, we transiently transfected wild-type or G309D c-Myc-tagged PINK1 cDNA constructs into monkey kidney COS-7 cells. The mutation did not alter production of mature full-length protein, which suggests it does not significantly affect protein stability (fig. S2). Both wild-type and mutant PINK1 localized to mitochondria, as assessed by immunofluoresence microscopy of transfected COS-7 cells (Fig.1) and human neuroblastoma SH-SY5Y cells (fig. S3). Furthermore, by probing with a c-Myc antibody, the mitochondrial localization of PINK1 was confirmed by Western blotting of mitochondrial enriched fractions obtained from COS-7 cells transiently transfected with c-Myc-tagged wild-type PINK1 cDNA (Fig.2).

We next investigated the effect of PINK1 mutations on mitochondrial function using a fluorescence-activated cell sorting (FACS)-based-assay of mitochondrial membrane potential $(\Delta \psi \mathrm{m})$ by examining the distribution of tetramethylrhodamine methyl ester (TMRM), a fluorescent lipophilic cation. Mitochondrial membrane potential is central to mitochondrial biology: it defines the transport of ions, including $\mathrm{Ca}^{2+}$ uptake, and provides the driving force for oxidative phosphorylation (6). SH-SY5Y cells were transiently co-transfected with wild-type and mutant PINK1 cDNA and a green fluorescent protein (GFP) reporter plasmid and then stressed with the peptide aldehyde, Cbz-leu-leuleucinal (MG-132), which inhibits the proteasome and induces apoptosis via distinct mechanisms including mitochondrial injury (7). Analysis of TMRM fluorescence in GFP-positive cells revealed that the PINK1 mutation had no significant effect on $\Delta \psi \mathrm{m}$ under basal conditions (Fig. 3A). However, following stress with MG-132 there was a significant decrease in $\Delta \psi \mathrm{m}$ from basal levels in cells transfected with G309D PINK1 compared with wild-type PINK1 (G309D, -44.1\% \pm 8.1 ; versus wild-type PINK1, $13.0 \% \pm 13.7 ; \mathrm{p}<0.01 ; \mathrm{n}=8$ ) (Fig. 3B; fig. S4).

We next determined the rate of apoptosis in MG-132stressed SH-SY5Y cells transfected with either wild-type or G309D PINK1 by FACS using Annexin V conjugated to the fluorochrome Phycoerythrin (Annexin V-PE). Annexin V has a high binding affinity for the membrane phospholipid, phosphatidylserine, that is exposed on the surface of apoptopic cells. Consistent with the changes in $\Delta \psi \mathrm{m}$ following stress, we found that over-expression of wild-type PINK1 but not mutant G309D significantly reduced the level of apoptopic cell death induced by MG-132 in GFP-positive cells (vector, $45.4 \% \pm 5.0$; wild-type $P I N K 1,32.7 \% \pm 4.0$; G309D, 45.8\% $\pm 5.0 ; \mathrm{p}<0.05$; ANOVA n = 12) (Fig. 3C; Fig. S5). Overall these preliminary findings suggest that wild type PINK1 may protect neurons from stress-induced mitochondrial dysfunction and stress-induced apoptosis and that this effect is abrogated by the G309D mutation.

Several lines of evidence suggest that impairment of mitochondrial activity could represent an early, critical event in the pathogenesis of sporadic PD (2). Environmental toxins such as 1-methyl-4-phenyl-1,2,3,6-tetrahydro-pyridin (MPTP) and the pesticide rotenone induce selective death of dopaminergic neurons through inhibition of complex I activity $(8,9)$. Complex I deficiency and a variety of markers of oxidative stress have been demonstrated in post-mortem brains of PD patients $(2,10,11)$. In addition, several reports have shown that mitochondrial dysfunction associated with oxidative stress can trigger $\alpha$-synuclein aggregation and accumulation although the exact mechanisms remain unclear (12).

The PINK1 mutations described here occur in the putative serine threonine kinase domain and thus conceivably could affect kinase activity or substrate recognition. Altered phosphorylation has been reported as a pathogenetic mechanism in other neurodegenerative diseases including Alzheimer's disease, tauopathy, and spinocerebellar ataxia $(13,14)$. The recent demonstration that phosphorylation of $\alpha-$ synuclein at serine 129 occurs in Lewy bodies in a variety of human synucleinopathy brains (15), suggests that altered phosphorylation may also play a role in PD. We hypothesize that PINK1 may phosphorylate mitochondrial proteins in response to cellular stress, protecting against mitochondrial dysfunction. PINK1 was originally shown to be upregulated by the tumor suppressor gene, PTEN, in cancer cells (5). In neurons the PTEN signaling pathway is involved in cell cycle 
regulation and cell migration and promotes excitotoxininduced apoptosis in the hippocampus (16). However, PINK1 has not been shown to exhibit any effects on PTENdependent cell phenotypes (5) and its role in the PTEN pathway therefore requires further investigation.

\section{References and Notes}

1. W. Dauer, S. Przedborski. Neuron 39, 889 (2003).

2. T.M. Dawson, V.L. Dawson. Science 302, 819 (2003).

3. E.M. Valente et al., Am. J. Hum. Genet. 68, 895 (2001).

4. E.M. Valente et al., Ann. Neurol. 51, 14 (2002).

5. M. Unoki, Y. Nakamura. Oncogene 20, 4457 (2001).

6. M.R. Duchen, A. Surin, J. Jacobson. Methods Enzymol. 361, 353 (2003).

7. J.H. Qiu et al., J. Neurosci. 20, 259 (2000).

8. W. J. Nicklas, I. Vyas, R.E. Heikkila. Life Sci. 36, 2503 (1985).

9. R. Betarbet et al., Nat. Neurosci. 3, 1301 (2000).

10. A.H. Schapira et al., Lancet 2, 1269 (1989).

11. P. Jenner, C.W. Olanow. Ann. Neurol. 44, S72 (1998).

12. T.B. Sherer et al., J. Neurosci. 22, 7006 (2002).

13. L. Buee, T. Bussiere, , V. Buee-Scherrer, A. Delacourte, P.R. Hof. Brain Res. Rev. 33, 95 (2000).

14. HK. Chen et al., Cell 113, 457 (2003).

15. H. Fujiwara et al., Nature Cell. Biol. 4, 160 (2002).

16. D.S. Gary, M.P. Mattson. Neuromolecular Med. 2, 261 (2002).

17. Supporting online material.

18. We thank the patients and families who participated in this study; J. Sinclair for technical assistance with FACS experiments, G. Howell for bioinformatic support, M. Duchen for useful discussion and Y. Nakamura and M. Unoki for PINK1 plasmid. Supported by grants from Telethon, Italy (E.M.V.), Italian Ministry of Health (E.M.V., B.D.), M.U.R.S.T. (B.D.), Parkinson's Disease Society, UK (N.W.W., D.S.L., R.J.H., D.G.H.), Brain Research Trust (P.M.A.S., N.W.W.), and the Deutsche Forschungsgemeinschaft (G.A., S.G.). M.M.K.M. is a Medical Research Council Clinical Research Training Fellow. GenBank accession numbers. PINK1 genomic sequence: AL391357. PINK1 mRNAs: AB053323, AF316873, AK075225, BC009534, BC028215. PINK1 protein: BAB55647, AAK28062, BAC11484, AAH09534, AAH28215.

\section{Supporting Online Material}

www.sciencemag.org/cgi/content/full/1096284/DC1

Materials and Methods

SOM Text

Figs $\mathrm{S} 1$ to $\mathrm{S} 5$

Tables S1 and S2

References
2 February 2004; accepted 1 April 2004

Published online 15 April 2004; 10.1126/science. 1096284

Include this information when citing this paper

Fig. 1. PINK1 is localized to the mitochondria in mammalian cells and its localization is not affected by the G309D mutation. (A-F) COS-7 cells transfected with wild-type (A to C) or G309D (D to F), c-myc-tagged PINK1 protein. Immunofluorescence was carried out with c-Myc antibody and mitotracker: c-myc-PINK1 [green $(\mathbf{A}, \mathbf{D})$ ]; mitotracker [red $(\mathbf{B}, \mathbf{E})]$; c-Myc-PINK1 and mitotracker merged $(\mathbf{C}, \mathbf{F})$.

Fig. 2. PINK1 is localized to the mitochondrial enriched fraction of mammalian cells. COS-7 cells were transiently transfected with c-Myc tagged wild-type PINK1. Cytoplasmic (lane 1) and mitochondrial enriched fractions (lane 2) were obtained and probed for c-Myc PINK1 expression by Western blot analysis using a c-Myc antibody. The same membrane was stripped and re-probed with Hsp60, complex I and GAPDH antibodies to determine the relative purity of fractions analyzed.

Fig. 3. Wild-type PINK1 but not mutant G309D PINK1 protects against stress induced mitochondrial dysfunction and apoptosis. (A,B) Effect of wild-type and mutant PINK1 on mitochondrial membrane potential $(\Delta \psi \mathrm{m})$ determined by FACS of GFP gated events of SH-SY5Y cells treated with vehicle (basal) or MG-132. (A) Basal TMRM values normalised to vector, mean \pm SEM percentage TMRM fluorescence of vector. (B) Mean \pm SEM percent change in median TMRM fluorescence from basal value for each

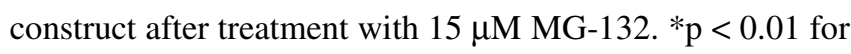
G309D versus wild-type PINK1; ANOVA with post-hoc Bonferroni correction; $\mathrm{n}=8$ from 3 independent experiments performed in duplicate or triplicate. (C) Effect of wild-type and mutant PINK1 on the amount of apoptosis determined by FACS of Annexin V-PE positive GFP gated events of SHSY5Y cells treated with vehicle (basal) or MG-132. Mean \pm SEM percent apoptopic cell death for each construct after treatment with vehicle or $15 \mu \mathrm{M}$ MG-132. *p $<0.05$; ANOVA, $\mathrm{n}=12$ from 4 independent experiments performed in triplicate. 
PINK1

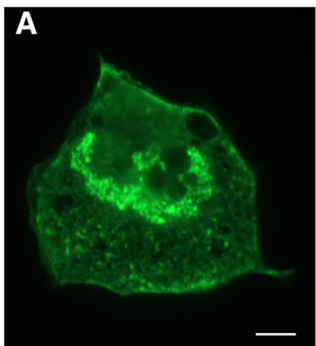

wild type

G309D
Mitotracker
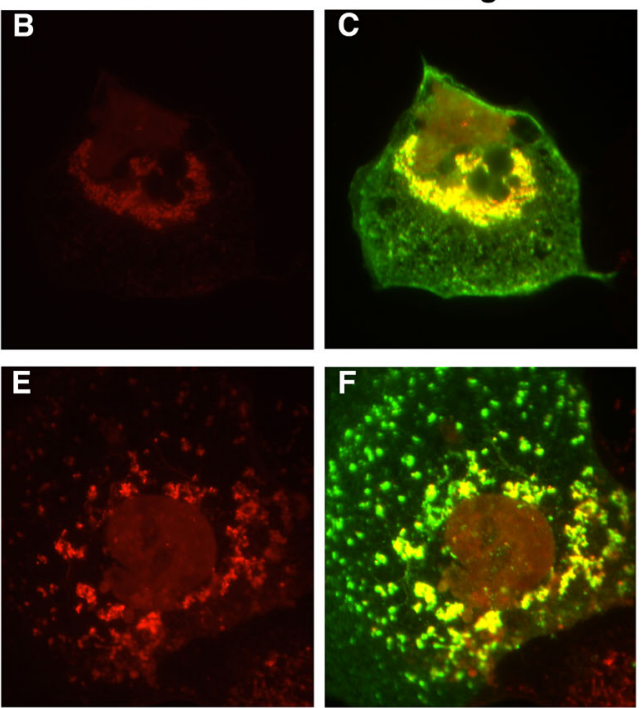

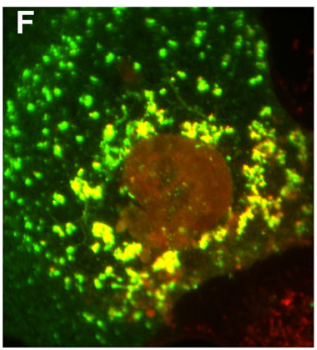




\section{$97 \mathrm{k}-$ $66 \mathrm{k}-$}

$45 \mathrm{k}$

$30 \mathrm{k}$ 


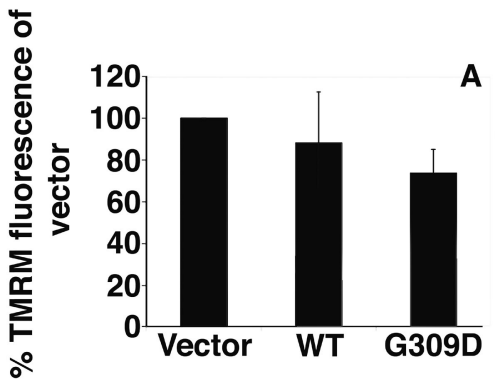

Plasmid
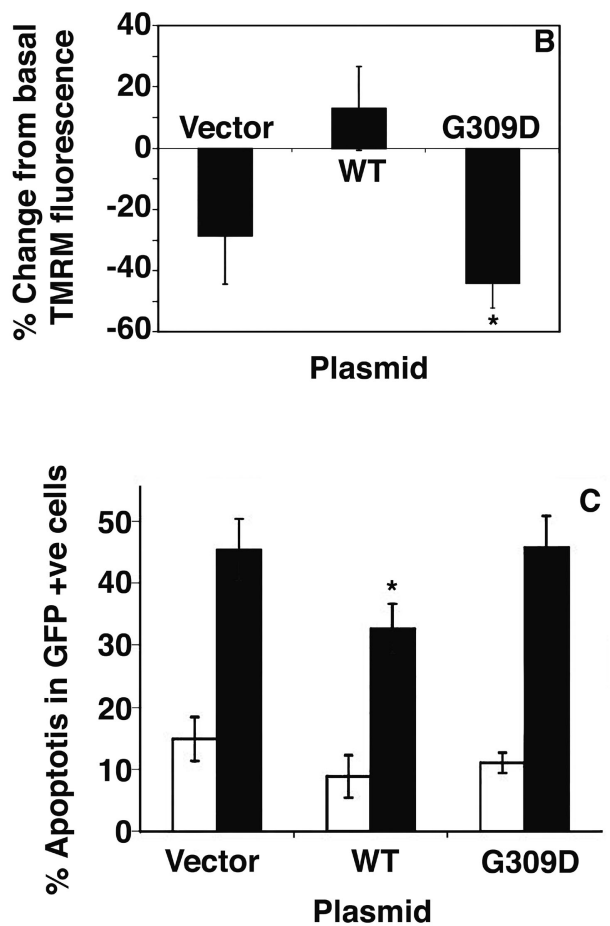